\title{
MODELAGEM E SIMULAÇÃO DA CAPTAÇÃO DE CÁLCIO POR ASTROGLIOMAS A PARTIR DE ESTÍMULOS GLUTAMATÉRGICOS
}

\author{
E. C. ZANATTA ${ }^{1}$, L. C. ASSIS ${ }^{2}$, A. F. BEM ${ }^{2}$ e L. T. PINTO ${ }^{1}$ \\ Universidade Federal de Santa Catarina, ${ }^{1}$ Departamento de Engenharia Química e \\ Engenharia de Alimentos, ${ }^{2}$ Departamento de Bioquímica - Florianópolis, SC \\ E-mail: elis_criz@yahoo.com.br e leonel@enq.ufsc.br
}

\begin{abstract}
A modelagem fenomenológica da dinâmica iônica nas sinapses neuronais é fundamental para o entendimento do processamento da informação cerebral. Parte desta dinâmica é controlada pela abertura de canais sensíveis a neurotransmissores, existentes nos neurônios e nas células gliais que contornam as sinapses. Neste trabalho foi desenvolvido um estudo experimental que resultou na proposta de modelo matemático para a descrição da dinâmica dos canais glutamatérgicos existentes nos astrócitos. O experimento, formado pelo uso de uma cultura de células de glioma C6, uma solução de cálcio e glutamato e um indicador de cálcio, foi realizado em um aparelho misturador de fluxo interrompido, Stopped-Flow Mixer, capaz de produzir dados de concentração de cálcio no interior das células, em intervalos de $10 \mathrm{~ms}$. A partir dos resultados foi desenvolvido modelo matemático para a dinâmica do cálcio através da membrana celular.
\end{abstract}

\section{INTRODUÇÃO}

Durante muito tempo acreditou-se que a sinapse era uma "conversa" restrita a dois neurônios; contudo, hoje se sabe que este fenômeno é muito mais complexo; envolvendo uma tríade, no qual também participa a célula glial astrocitária - fenômeno este popularmente conhecido como sinapse tripartite (Fields \& Graham, 2002; Nimmerjahn, 2009). Segundo Araque e Navarrete (2010), numerosos estudos foram realizados acerca de tal temática durante os últimos anos; e, por meio deles, pode-se acreditar na existência de uma sinalização bidirecional entre astrócitos e neurônios. Wade et al (2011), por sua vez, corroboram com tal afirmação ao salientarem que embora as células astrocitárias não possam provocar a propagação de potenciais de ação, elas também podem se comunicar fazendo isto de forma bidirecional com outros astrócitos e neurônios através da liberação de transmissores e da propagação de ondas de cálcio. Os autores ainda reforçam que a interação dos receptores de membrana presentes nos astrócitos com o neurotransmissor glutamato conduz imediatamente a uma elevação transitória nos níveis intracelulares de cálcio astrocístico, representando, deste modo, um dos meios mais conhecidos de excitação em astrócitos. Como resposta a elevação de cálcio, os astrócitos acabam por responder a tal estímulo liberando diferentes substâncias neuroativas: os gliotransmissores. Os gliotransmissores, por sua vez, são os responsáveis pela modulação da transmissão sináptica, agindo tanto nos receptores pré como nos pós-sinápticos. Por meio deste trabalho pretendese elucidar de que modo ocorre o processo cinético de captação de cálcio por células gliais, mais precisamente os astrogliomas, a partir de um estímulo glutamatérgico, como ocorrem nas sinapses tripartites. A realização deste trabalho procura englobar não somente a visão da engenharia química frente a um processo fisiológico, mas na realidade unir conhecimentos multidisciplinares; visando, deste modo, construir um entendimento uniforme da temática. $\mathrm{O}$ 
estudo de tal temática ganha força dada a dificuldade de encontrar referências literárias; principalmente no que tange a dados numéricos extraídos de metodologias experimentais. Reforçando a relevância de se realizar tal estudo, Goldberg et al (2010), lembram que ainda hoje vários aspectos da sinalização de cálcio em astrócitos permanecem por serem elucidados.

\section{MATERIAIS E MÉTODOS}

A parte experimental do trabalho foi realizada com o auxílio de um equipamento capaz de analisar reações de rápida cinética, o Stopped Flow Mixer 4000/S, SFM-4000/S, da BioLogic Science Instruments. Ele é um instrumento de fluxo interrompido que permite trabalhar-se com reações químicas de cinéticas muito rápidas. Nestes equipamentos, têm-se pequenos volumes de soluções sendo rapidamente conduzidas a partir de seringas para um misturador de elevada eficiência para que se inicie uma reação rápida. A substância resultante desta reação é então deslocada até uma célula de observação (cubeta) onde passa pela análise, que pode ser por fluorescência, absorbância e dispersão de luz. Muitos processos biológicos ocorrem em uma faixa de tempo de milissegundos; o que requer aparatos rápidos o suficiente para medir suas dinâmicas. O SFM-4000/S especificamente apresenta um subsistema mecânico constituído por quatro seringas independentes; o que permite que de duas a quatro soluções distintas possam ser misturadas e injetadas na cubeta de observação (BioLogic Science Instruments, 2012).

Foram utilizadas células de glioma C6 (astrogliomas) para formar a cultura utilizada no trabalho. Gliomas são os tumores primários mais comuns nos tecidos cerebrais e representam uma das linhagens celulares tumorais da glia que possuem propriedades oliodendrocitárias tão boas quanto às de seu astrócito progenitor. $\mathrm{O}$ neurotransmissor escolhido foi o L-glutamato, que é o principal neurotransmissor excitatório do sistema nervoso. $\mathrm{O}$ cálcio foi obtido por meio de uma solução de cloreto de cálcio. Como indicador de cálcio, foi usado o FURA-2/AM, principalmente porque o éster do acetoximetil (AM) da sonda fluorescente FURA-2 é permeável às células, não necessitando a utilização de métodos invasivos (Lambert, 1999; Simpson, 1999).

Cultura de células: As células ficaram acondicionadas em placas contendo meio de cultura, em estufa à $37^{\circ} \mathrm{C}$ e $5 \%$ de dióxido de carbono. Após retirando-se e descartando-se o meio de cultura a que elas estavam acondicionadas. Após esta etapa, acrescentou-se $1,8 \mathrm{~mL}$ de tampão fosfato salino (PBS) - para lavar as células - e $200 \mu \mathrm{L}$ de tripsina - para que houvesse o desprendimento das células do fundo da placa. Procedeu-se a incubação das células durante três minutos, visando facilitar o processo de desprendimento celular. Ao término desta etapa, adicionou-se $2 \mathrm{~mL}$ de meio de cultura e se procedeu a homogeinização da suspensão celular, sendo as células levadas a centrífuga a $1,2 \mathrm{rpm}$ durante quatro minutos. Ao ser retirada da centrifuga, o sobrenadante foi descartado e o pellet celular ressuspenso em $1 \mathrm{~mL}$ de meio de cultura. Se da continuidade ao tratamento das células realizando a contagem das mesmas em Câmara de Neubauer $(10 \mu \mathrm{L}$ de célula $+190 \mu \mathrm{L}$ de azul de tripan). Após esta etapa, realocaram-se as células em placa de petri contendo $8 \mathrm{~mL}$ de meio de cultura, e procedeu-se ao repique celular $-200 \mu \mathrm{L}$ de células em $7 \mathrm{~mL}$ de meio por placa de cultura.

Após o tratamento/plaqueamento das células: (1) Preparou-se uma suspensão das células (em PBS); (2) Preparou-se 1mM de solução-estoque com FURA-2/AM em DMSO; (3) Diluiu-se uma alíquota do indicador (solução-estoque) de 100 a 500 vezes em PBS; (4) 
Adicionou-se a solução aquosa do indicador para um volume igual de células; (5) Incubouse durante 30 minutos a $37^{\circ} \mathrm{C}$; (6) Lavou-se as células duas vezes com PBS; (7) As células foram suspensas novamente em PBS e acondicionadas em seringas de $10 \mathrm{~mL}$.

Preparação da solução contendo cálcio: A solução contendo cálcio foi preparada a partir de cloreto de cálcio. Adotando-se, como padrão a concentração de 5M. A solução foi diluída com água Milli-q e acondicionada em seringas de $10 \mathrm{~mL}$.

Preparação do glutamato: Diluição com água Milli-q para se chegar a concentração de $2 \mu \mathrm{M}$, uma vez que em condições fisiológicas, a concentração deste neurotransmissor na

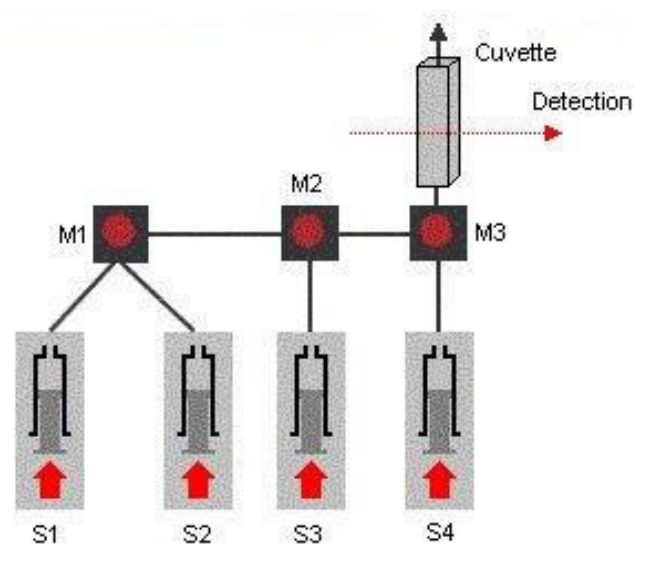

Figura 1. Mecanismo de aquisição de dados empregado pelo SFM-4000/S. Fonte: Biologic Science Instruments (2012) fenda sináptica fica em torno deste valor.

Processo de mensuração da captação de cálcio por células de glioma C6: Após o preparo das células e carregamento das mesmas com indicador de cálcio, ajustou-se o equipamento para a aquisição de dados por fluorescência. Os comprimentos de onda de excitação utilizados foram $340 \mathrm{~nm}$ e $380 \mathrm{~nm}$; com comprimento de onda de detecção de $515 \mathrm{~nm}$ (conseguido através do uso de um filtro de corte instalado no tubo fotomultiplicador). As mensurações foram realizadas em temperatura ambiente $\left(22^{\circ} \mathrm{C}\right)$, sendo a mesma controlada por condicionador de ar. A análise foi realizada em um tempo de 3 segundos.

\section{MODELAGEM MATEMÁTICA}

O indicador de cálcio FURA-2 só emite fluorescência após interação com cálcio se iluminado a um determinado comprimento de onda. Entretanto, fluorescência não é sinônimo de concentração de cálcio, e, por isso, se fez necessário um estudo mais aprofundado dos fenômenos envolvidos na reação, de modo a permitir a conversão das unidades/valores. Há na literatura uma equação proposta por Grynkiewicz e colaboradores no ano de 1985 que realiza a conversão da fluorescência adquirida para concentração de cálcio intracelular.

$$
\mathrm{C}=\frac{[\mathrm{CaT} \cdot \mathrm{S}]}{[\mathrm{S}]} \cdot \mathrm{k} \cdot \frac{(\mathrm{R}-\mathrm{Rmin})}{(\mathrm{Rmax}-\mathrm{R})}
$$

Onde:

CaT (Cálcio Total); C (Concentração de cálcio citosólico); R (Fluorescência emitida com glutamato); Rmax (Fluorescência máxima obtida); Rmin (Fluorescência mínima obtida); S (Fator de escala, determinado a um comprimento de onda de $380 \mathrm{~nm}$ ).

Para a obtenção da curva de fluorescência mínima, Rmin, produzida com níveis reduzidos de cálcio, foi utilizado o detergente enzimático TRITON X-100 (concentração de 0,1\%), adicionado à solução com células impregnadas com FURA-2. Após um período de 4 minutos a solução resultante foi considerada livre da presença de células. Esta solução foi 
então adicionada à cubeta, junto com uma solução de cálcio e outra com o composto EGTA, ou ácido tetracético etilenoglicol (concentração de 4,5mM). As principais reações que ocorrem na solução são as seguintes:

$\mathrm{Ca}^{2+}+$ Fura $2 \stackrel{k_{1}}{\rightarrow}[\text { Ca: Fura2 }]^{2+}$

$C a^{2+}+E G T A \stackrel{k_{2}}{\rightarrow}$ EGTA. $C a^{2+}$

Onde Ca, Fura2, Fura2.Ca, EGTA e EGTA.Ca são as concentrações molares. EGTA e FURA-2 competem pelo cálcio, com a prevalência do primeiro. Em função da alta afinidade do EGTA pelo cálcio, sua concentração fica próxima de zero, reduzindo ao mínimo a ocorrência da reação com o FURA-2. Para corroborar isto, BERGH e colaboradores (1995) obtiveram, em condições semelhantes as existentes no presente trabalho, para as constantes cinéticas os seguintes valores: $\mathrm{k} 1=5,0 \times 10^{10} \mathrm{M}^{-1} \mathrm{~s}^{-1} ; \mathrm{k} 2=3,2 \times 10^{7} \mathrm{~s}^{-1}$. A diferença de três ordens de grandeza entre as constantes cinéticas reforçam a consideração de alta afinidade do EGTA pelo Cálcio.

Para a curva de fluorescência máxima, Rmax, o detergente enzimático TRITON X-100 é adicionado à cubeta, junto com a solução de cálcio e a solução de células impregnadas com FURA-2. A medida que o TRITON destrói as células, o FURA-2 e o Cálcio reagem, conforme o mecanismo de reação (2), resultando em uma cinética de segunda ordem:

$\frac{d C a}{d t}=-k_{1}$. Ca.Fura2

Finalmente, no experimento com adição de glutamato, na cubeta são adicionadas simultaneamente as soluções com cálcio, células impregnadas com FURA-2 e glutamato. Para a ocorrência da reação (2) é necessário que o glutamato abra os canais e que o cálcio penetre nas células.

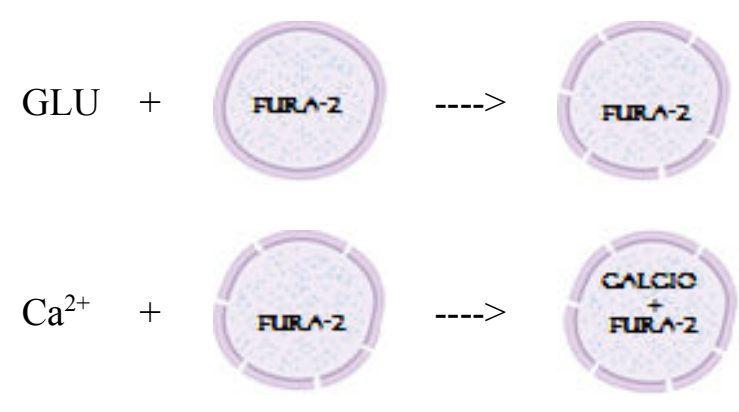

O cálcio penetra nas células por difusão. Considerando os dois meios em contato com a membrana celular homogêneos, é possível escrever o fluxo através dos canais por um modelo tipo Linear Drive Force.

$\frac{d C a_{i n}}{d t}=-n \cdot A_{c} \cdot \frac{1}{\tau} \cdot\left[C a_{\text {in }}-C a_{o u t}\right]$

Onde in e out se referem à concentração de cálcio dentro e fora da célula, $\boldsymbol{A c}$ é a área máxima, correspondendo a situação em que todos os canais estão abertos, $\tau$ é a espessura da membrana e $\boldsymbol{n}$ uma função que define a fração de canais abertos em um dado momento. Neste trabalho, $\boldsymbol{n}$ é calculado por uma Distribuição Normal, dependente da concentração de glutamato. 
$n(t)=\frac{G l u}{\sqrt{2 \pi}} e^{\left(-\frac{t^{2}}{2}\right)}$

\section{RESULTADOS E DISCUSSÃO}

Os resultados experimentais são apresentados nas figuras 2-4.

Da figura 4 pode-se observar que em todos os experimentos ocorreu captação de cálcio pelas células. Os valores negativos iniciais ocorrem porque no início da reação a

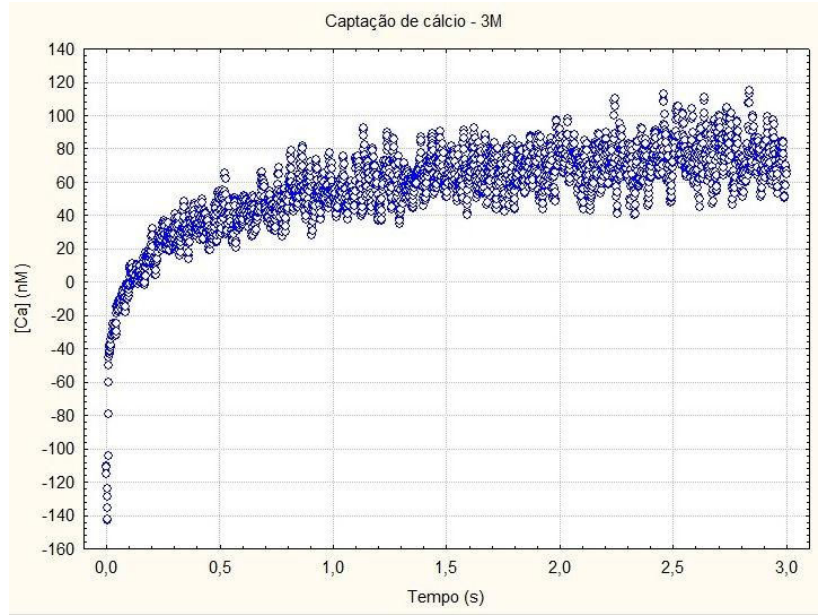
fluorescência mínima é maior do que a fluorescência com glutamato. Nestas condições a aplicação da equação 1 leva aos valores negativos iniciais. $\mathrm{O}$ fato de $\mathrm{o}$ indicador ser raciométrico reforça a justificativa aos resultados negativos encontrados, uma vez que, se trabalhou com um fluorocromo, ou seja, moléculas químicas que absorvem luz em determinado comprimento de onda (luz ultravioleta) e emitem em outro (luz visível); isto é; ficam fluorescentes (Tsien, 2010).

Figura 2. Captação de $\mathrm{Ca} 2+$ ao longo do tempo para uma concentração de cálcio de $3 \mathrm{M}$.



A metodologia empregada neste experimento é baseada em espectrofluorimetria. O seu emprego se dá no intuito de analisar justamente a fluorescência de uma amostra. Mendes (s.d.) lembra que o conhecimento da absorção de luz pela matéria é a forma mais usual de determinar a concentração de compostos presentes em solução, sendo a intensidade da cor de uma solução proporcional à concentração das moléculas absorventes de luz.

Figura 3. Captação de Ca2+ ao longo do tempo para uma concentração de cálcio de 5M.

A absorção da luz pela matéria envolve a incorporação da energia contida no fóton pela estrutura das moléculas absorventes (neste caso o FURA-2). Quando isso acontece, as moléculas absorventes passam do estado fundamental (estado energético mais baixo) para o estado excitado (estado energético mais alto). A absorção da luz deixa o sistema energizado, podendo ocorrer desde uma simples excitação, até a ionização da matéria. "Se o elétron apenas oscila, sem deixar seu orbital, o sistema apenas se aquece. Nos movimentos moleculares também se observa aquecimento do sistema". A energia de elétrons repostos na 
posição inicial é emitida como fluorescência ou fosforescência (Vines et al., 2012).

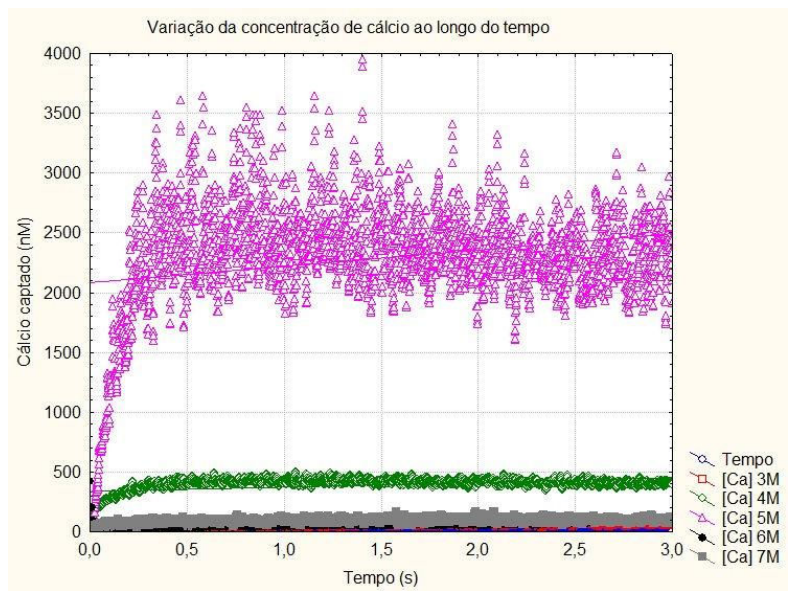

Figura 4. Captação de $\mathrm{Ca} 2+$ ao longo do tempo para todas as concentrações de cálcio.

Por fim, para uma análise geral dos resultados obtidos, sem os valores negativos iniciais, um gráfico contendo todas as concentrações de cálcio juntas se faz interessante.

O gráfico acima demonstra que na concentração de cálcio de $5 \mathrm{M}$ houve uma captação de cálcio muito mais significativa do que nas demais. Concentração esta, segundo André (2004), tida como fisiológica para o meio extracelular. Abaixo desta concentração, as células aparentemente foram inibidas pela falta de substrato; já acima disto, as células foram inibidas pelo excesso do mesmo. $\mathrm{O}$ excesso de substrato realmente pode proporcionar uma saturação do captador do substrato (no caso em questão, as células), reduzindo assim sua atividade.

Seguida a concentração de $5 \mathrm{M}$, a concentração de cálcio que foi mais bem captada pelas células foi a de $4 \mathrm{M}$, como pode ser observado no gráfico acima apresentado. $\mathrm{Na}$ concentração de $3 \mathrm{M}$ houve menos captação do que na de $4 \mathrm{M}$, o que vai ao encontro do explicitado acima; já na concentração de $7 \mathrm{M}$ captou-se menos cálcio do que na de $6 \mathrm{M}$, o que comprova haver inibição por excesso de substrato.

\section{CONCLUSÃO}

Possuindo trilhões de células; cada uma com milhares receptores de membrana; parece difícil acreditar que o corpo humano consiga trabalhar de forma tão harmônica e manter um estado de equilíbrio. Contudo, esta tarefa somente se torna possível, graças à existência de um complexo sistema de comunicação celular. A sinalização celular tem por intuito controlar e coordenar as mais diversas atividades e funções celulares. Entre os sinalizadores existentes no corpo humano, o cálcio é um dos que mais merecem destaque; dado o seu envolvimento na maior parte dos processos celulares. $O$ cálcio se liga a milhares de proteínas, fazendo com que haja alterações tanto na localização proteica, quanto na associação ou mesmo na função. Os experimentos mostram a importância da intervenção do íon cálcio para as mais diversas respostas fisiológicas.

\section{AGRADECIMENTOS}

Os autores agradecem à CAPES e ao CNPq pelo apoio a este trabalho, na forma de bolsa de estudos para os primeiros dois autores e recursos para a aquisição do SFM-4000/S, dentro do Edital Pró-Equipamentos de 2011.

\section{REFERÊNCIAS}

ANDRÉ, E. Evidências sobre o envolvimento dos receptores vanilóides nas ações biológicas produzidas pelos sesqui-terpenos poligodial e drimanial. 2004. 85 f. Tese em Farmacologia. Universidade Federal de Santa Catarina, Florianópolis. 
ARAQUE, A; NAVARRETE, M., Glial cells in neuronal network function. Rev. Philosophical Transactions of the Royal Society B, vol. 365, p. 2375-2381, 2010.

BERGH, V.V. et al., Photophysics of the fluorescent $\mathrm{Ca}^{2+}$ Indicator Fura-2, Biophysical Journal, 68, 1110-1119, 1995

DANI, J. W.; CHERNJAVSKY, A; SMITH, S., Neuronal Activity Triggers Calcium Waves in Hippocampal Astrocyte Network. Rev. Neuron, vol 8, p. 429-440, 1992.

FIELDS, R. D \& GRAHAM, B. S., New Insights into Neuron-Glia Communication. Science, 298 (5593), 556-562, 2002.

GOLDBERG,M; PITTA, M; VOLMAN, V; BERRY, H; BEN-JACOB,E. Nonlinear Gap Junctions Enable Long-Distance Propagation of Pulsating Calcium Waves in Astrocyte Networks Rev. PLOS Computational Biology, vol 6, 2010.

GRYNKIEWICZ, G; POENIE, M; TSIEN, R.Y., A new generation of $\mathrm{Ca}^{2+}$ Indicators with Greatly Improved Fluorescence Properties. The Journal of Biological Chemistry, vol. 260, no6, p. 3440-3450, 1985.

LAMBERT, D.G. Signaling protocols. Editora Humana Press, 1999.

NIMMERJAHN, A., Astrocytes going live: advances and challenges. Rev. the Journal of Physiology, vol.587, p. 1639-1647, 2009.

SIMPSON, A.W.M. Fluorescent Measurement of $\left[\mathrm{Ca}^{2+}\right]_{\mathrm{c}}$. In: LAMBERT, D.G. Calcium Signaling Protocols. Vol. 114. Ed. Human Press. 1999. Cap. 01, p. 03-25.

TSIEN, R.Y., Fluorescence readouts of biochemistry in live cells and organisms. In: Molecular Imaging: Principles and Practice, ed. WEISSLEDES, et al., People's Medical Publishing House - USA. Cap. 48, p. 808-828, 2010.

VINES, A; McBEAN, G. J; FERNANDEZ, A. B., Continuous Measurement of Intracellular calcium on the BD Accuri ${ }^{\mathrm{TM}}$ C6 Flow cytometer. In: BD Biosciences, 2012, p.01-12.

WADE, J.J; MCDAID, L.J; HARKIN, J; CRUNELLI, V; KELSO, J.A., Bidirectional coupling between astrocytes and neurons mediates learning and dynamic coordination in the brain: a multiple modeling approach. PLOS ONE, vol. 6; nº 12, p.01-24, 2011. 